Original Research Paper

\title{
q-Euler Lagrange Equation
}

\author{
${ }^{1,2}$ Amna Hasan, ${ }^{3,4}$ Hakeem A. Othman and ${ }^{5,6}$ Sami H. Altoum \\ ${ }^{I}$ Department of Mathematics, College of Sciences, Albaha University, KSA, Saudi Arabia \\ ${ }^{2}$ Department of Mathematics, Al Neelain University, Sudan \\ ${ }^{3}$ Department of Mathematics, AL-Qunfudhah University College, Umm Al-Qura University, Mecca, KSA, Saudi Arabia \\ ${ }^{4}$ Department of Mathematics, Rada' a College of Education and Science, University Albaydha, Albaydha, Yemen \\ ${ }^{5}$ AL-Qunfudhah University College, Umm Al-Qura University, Mecca. KSA, Saudi Arabia \\ ${ }^{6}$ Academy of Engineering Sciences, Khartoum, Sudan
}

Article history

Received: 03-10-2019

Revised: 04-11-2019

Accepted: 22-11-2019

Corresponding Author:

Hakeem A. Othman

Department of Mathematics,

AL-Qunfudhah University

College, Umm Al-Qura

University, Mecca, KSA and

Department of Mathematics, Rada'a College of Education and Science, University

Albaydha, Albaydha, Yemen

Email: hakim_albdoie@yahoo.com

\section{Introduction}

The calculus of variation is a one of the most important division of classical mathematical analysis as regards application. The aim of this study is to supply the reader with a certain minimum of problems covering the basic divisions of classical calculus of variation. In Euler; s methods, the values of the functional see Equation 1 are considered not on arbitrary curves admissible in the given variational problem, but only on polygonal curves composed of a given number $\mathrm{n}$ of straight-line segments with specified abscissas of the vertices. If we have a certain class $M$ of function $y(x)$ and each function $y \in M$ there is associated, by some law, a definite number $J$, then we say a functional $J$ is defined in the class $M$ and we write $J=J[y(x)]$. The class $M$ of function $y(x)$ on which the functional $J[y(x)]$ is called the definition of functional. In mathematics, a q-analog of a theorem identity or expression is a generalization involving a new parameter $q$ that returns the original theorem, identity or expression in the limit as $q \rightarrow 1$, see (Altoum, 2018a; 2018b; Rguigui, 2015a; 2015b; Bangerezako, 2004).

The Euler-Lagrange D.E is the essential equation of variational principle. It is defined by an integral of the form:
$J=\int f\left(t, y, y^{\prime}\right) d t$

Where:

$$
y^{\prime}=\frac{d y}{d t}
$$

Then $J$ has a stationary value if the Euler-Lagrange differential equation:

$$
\frac{\partial f}{\partial y}-\frac{d}{d t}\left(\frac{\partial f}{\partial y^{\prime}}\right)=0
$$

is satisfied. If time-derivative notation is replaced instead by space-derivative notation, the equation becomes:

$$
\frac{\partial f}{\partial y}-\frac{d}{d x}\left(\frac{\partial f}{\partial y_{x}}\right)=0
$$

The Euler-Lagrange differential equation is implemented as Euler equations $[f, u[x], x]$ in the Wolfram Language package variational methods. In many physical problems, the partial derivative of with 
respect to turns out to be 0 , in which case a manipulation of the Euler-Lagrange differential equation reduces to the greatly simplified and partially integrated form known as the Beltrami identity:

$$
f-y_{x} \frac{\partial f}{\partial y_{x}}=c
$$

For three independent variables, the equation generalizes to:

$$
\frac{\partial f}{\partial u}-\frac{\partial}{\partial x} \frac{\partial f}{\partial u_{x}}-\frac{\partial}{\partial y} \frac{\partial f}{\partial u_{y}}-\frac{\partial}{\partial z} \frac{\partial f}{\partial u_{z}}=0
$$

This paper organized as follows: Section 1 and 2 introduce the basic concepts of the study. In next section, we present an analogous of the classical Euler Lagrange equation, finally we deduce some theorems.

\section{Preliminaries}

We introduce the q-Derivative, we recall some basic notations used in q-calculus. The natural number $\mathrm{n}$ has the following $q$ deformation:

$$
[n]_{q}=1+q+q^{2}+\cdots+q^{n-1}, \text { with }[0]_{q}=0 .
$$

Occasionally, we shall write $[\infty]_{q}$ for the limit of these numbers: $\frac{1}{(1-q)}$. The $q$ factorials and $q$ binomial coefficients are defined naturally as:

$$
[n]_{q} !=[1]_{q} \cdot[2]_{q} \cdot[3]_{q} \cdots[n]_{q},
$$

With:

$$
[0]_{q}=0
$$

Here is a decent redirection for any individual who knows what the derivative of a simple function is $f(x)$. The modern theory of differential and integral calculus began in the 20th century with the works of Newton and Leibniz. As it is well known, see (Altoum et al., 2017), the derivative of a function $f(x)$ w.r.t the variable $x$ is by definition:

$$
f^{\prime}(x)=\lim _{h \rightarrow 0} \frac{f(x+h)-f(x)}{h} .
$$

Now, let us consider the following expression:

$$
f^{\prime}(x)=\lim _{q \rightarrow 1} \frac{f(q x)-f(x)}{q x-x} .
$$

Of course, this is not valid when $q=1$ or $x=0$ but otherwise this alternative formula is equivalent to the usual derivative. You can convince yourself by writing $\frac{f(x+(q-1) x)-f(x)}{(q-1) x}$, the term $(q-1) x$ playing the role of $h$.

At the beginning of the 20th century, F.H. Jackson studied this modified derivative and many of its consequences, see (Rguigui, 2016a; 2016b; Rguigui, 2018a; 2018b). The key concept is the q-derivative operator defined as follows when $0<q<1$ :

$$
\left(D_{q} f\right)_{q \rightarrow 1}(x)=\lim _{q \rightarrow 1} \frac{f(q x)-f(x)}{q x-x} .
$$

This q-derivative can be applied to functions not containing 0 in their domain of definition. Then it reduces to the ordinary derivative when $q$ goes to:

$$
\lim _{q \rightarrow 1}\left(D_{q} f\right)(x)=f^{\prime}(x)
$$

As an example, we compute the q-derivative of $x^{2}$ $+2 x+1$ :

$$
D_{q}\left(x^{2}+2 x+1\right)=\frac{\left[(q x)^{2}+2(q x)+1\right]-\left[x^{2}+2 x+1\right]}{q x-x} .
$$

One can easily check that the q-derivative operator is linear:

$$
\begin{aligned}
& D_{q}(f+g)=D_{q} f+D_{q} g D_{q} \\
& \left(D_{q} \lambda(f)=\lambda\left(D_{q}(f),\right.\right.
\end{aligned}
$$

the product rule is slightly modified but it approaches the usual product rule when $q$ goes to one:

$$
\left(D_{q}(f g)\right)(x)=f(q x)\left(D_{q} g\right)(x)+\left(D_{q} f\right)(x) g(x) .
$$

\section{q-Euler Lagrange Equation}

As analogous of the classical Euler Lagrange equation:

$\frac{\partial f}{\partial y}-\frac{d}{d t}\left(\frac{\partial f}{\partial y^{\prime}}\right)=0$

Where:

$$
y^{\prime}=\frac{d y}{d t}
$$

We introduce the q-Euler Lagrange equation as follows: 


$$
D_{q, y} f-D_{q, t}\left(D_{q, y_{q}^{\prime}} f\right)=0
$$

Where:

$$
\begin{aligned}
& f=f\left(t, y, y_{q}^{\prime}\right) \\
& y_{q}^{\prime}(t)=D_{q, t} y(t)=\frac{y(t)-y(q t)}{t(1-q)} \\
& D_{q, y} f\left(t, y(t), y_{q}^{\prime}(t)\right)=\frac{f\left(t, y(t), y_{q}^{\prime}(t)\right)-f\left(t, y(t), y_{q}^{\prime}(t)\right)}{y(t)(1-q)}
\end{aligned}
$$

$$
D_{q, y_{q}^{\prime}} f=\frac{f\left(t, y(t), y_{q}^{\prime}(t)\right)-f\left(t, y(t), y_{q}^{\prime}(q t)\right)}{y_{q}^{\prime}(t)(1-q)} .
$$

From the above discussion, we obtain the following theorem.

\section{Theorem 3.1}

Let 1 given by:

$$
\begin{gathered}
\left(\frac{f\left(t, y(t), y_{q}^{\prime}(t)\right)-f\left(t, y(q t), y_{q}^{\prime}(t)\right)}{y(t)(1-q)}\right)-\frac{l(t)-l(q t)}{t(1-q)}=0 \\
\left(\frac{f\left(q t, y(q t), y_{q}^{\prime}(q t)\right)-f\left(q t, y\left(q^{2} t\right), y_{q}^{\prime}(q t)\right)}{y(q t)(1-q)}\right)-\frac{l(q t)-l\left(q^{2} t\right)}{q(1-q) t}=0 \\
\vdots \\
\left(\frac{f\left(\left(q^{n-1} t\right), y\left(q^{n-1} t\right), y_{q}^{\prime}\left(q^{n-1} t\right)\right)-f\left(\left(q^{n-1} t\right), y\left(q^{n} t\right), y_{q}^{\prime}\left(q^{n-1} t\right)\right)}{y\left(q^{n-1} t\right)(1-q)}\right)-\frac{l\left(q^{n-1} t\right)-l\left(q^{n} t\right)}{q^{n-1}(1-q) t}=0 .
\end{gathered}
$$

Then, we obtain:

$$
\begin{gathered}
\left(\frac{f\left(t, y(t), y_{q}^{\prime}(t)\right)-f\left(t, y(q t), y_{q}^{\prime}(t)\right)}{y(t)}\right)-\frac{l(t)-l(q t)}{t}=0 \\
q\left(\frac{f\left(q t, y(q t), y_{q}^{\prime}(q t)\right)-f\left(q t, y\left(q^{2} t\right), y_{q}^{\prime}(q t)\right)}{y(q t)}\right)-\frac{l(q t)-l\left(q^{2} t\right)}{t}=0 \\
\vdots \\
q^{n-1}\left(\frac{f\left(\left(q^{n-1} t\right), y\left(q^{n-1} t\right), y_{q}^{\prime}\left(q^{n-1} t\right)\right)-f\left(\left(q^{n-1} t\right), y\left(q^{n} t\right), y_{q}^{\prime}\left(q^{n-1} t\right)\right)}{y\left(q^{n-1} t\right)}\right)-\frac{l\left(q^{n-1} t\right)-l\left(q^{n} t\right)}{t}=0
\end{gathered}
$$

This leads to:

$$
\sum_{k=0}^{n-1} q^{k}\left\{\frac{f\left(q^{k} t, y\left(q^{k} t\right), y_{q}^{\prime}\left(q^{k} t\right)\right)-f\left(q^{k} t, y\left(q^{k+1} t\right), y_{q}^{\prime}\left(q^{k} t\right)\right)}{y\left(q^{k} t\right)}\right\}=\frac{l(t)-l\left(q^{n} t\right)}{t} .
$$


Which completes the proof.

\section{Remark 1}

Using Theorem 3.1, for $n \rightarrow$ 1, the q-Euler Lagrange Equation (3) is equivalent to:

$$
\begin{aligned}
& \sum_{k=0}^{\infty} q^{k}\left\{\frac{f\left(q^{k} t, y\left(q^{k} t\right), y_{q}^{\prime}\left(q^{k} t\right)\right)-f\left(q^{k} t, y\left(q^{k+1} t\right), y_{q}^{\prime}\left(q^{k} t\right)\right)}{y\left(q^{k} t\right)}\right\} \\
& =\frac{l(t)}{t} .
\end{aligned}
$$

\section{Examples}

Recall that, the classical standard example, for $f$ given by:

$$
f\left(t, y, y^{\prime}\right)=\sqrt{1+y^{\prime 2}}
$$

We get:

$$
y=A t+B
$$

That is, the function must have constant first derivative and thus it is graph is a straight line.

Now, we will study the q-analogue of this standard example.

\section{Theorem 4.1}

Let $\mathrm{f}$ given by:

$$
f\left(t, y_{q}, y_{q}^{\prime}\right)=\sqrt{1+y_{q}^{\prime 2}}
$$

Satisfying the q-Euler Lagrange equation:

$$
D_{q, y} f-D_{q, t}\left(D_{q, y_{q}^{\prime}} f\right)=0 .
$$

Then, we get $y_{q}=A t+C$, for constants A and C.

\section{Proof}

Using equation (5), we obtain:

$$
D_{q, y_{q}^{\prime}} f=\frac{\sqrt{1+y_{q}^{\prime 2}}-\sqrt{1+q y_{q}^{\prime 2}}}{y_{q}^{\prime}(1-q)} .
$$

Since, we have:

$$
D_{q, y} f=0
$$

Then, by Equation (7), we get:

$$
D_{q, t}\left(D_{q, y_{q}^{\prime}} f\right)=0
$$

Therefore, we obtain:

$$
D_{q, y^{\prime}} f=c,
$$

Then, we get:

$$
\frac{\sqrt{1-y_{q}^{\prime 2}}-\sqrt{1+q y_{q}^{\prime 2}}}{y_{q}^{\prime}(1-q)}=c .
$$

This gives:

$$
\begin{gathered}
\sqrt{1+y_{q}^{\prime 2}}-\sqrt{1+q y_{q}^{\prime 2}}=c y_{q}^{\prime}(1-q) \\
\sqrt{1+q y_{q}^{\prime 2}}-\sqrt{1+q^{2} y_{q}^{\prime 2}}=c q y_{q}^{\prime}(1-q) \\
\vdots \\
\sqrt{1+q^{n-1} y_{q}^{\prime 2}}-\sqrt{1+q^{n} y_{q}^{\prime 2}}=c q^{n-1} y_{q}^{\prime}(1-q) .
\end{gathered}
$$

From which we get:

$\sqrt{1+y_{q}^{\prime 2}}-\sqrt{1+q^{n} y_{q}^{\prime 2}}=c q^{n-1} y_{q}^{\prime}(1-q)+\ldots .+c y_{q}^{\prime}(1-q)$.

Then, we deduce:

$$
\begin{aligned}
& \sqrt{1+y_{q}^{\prime 2}}-\sqrt{1+q^{n} y_{q}^{\prime 2}}=c(1-q) y_{q}^{\prime} \sum_{k=0}^{n-1} q^{k} \\
& =c(1-q) y_{q}^{\prime}\left(\frac{1-q^{n}}{1-q}\right) \\
& =c[n]_{q}(1-q) y_{q}^{\prime} .
\end{aligned}
$$

As $n \rightarrow \infty$, we obtain:

$$
\sqrt{1+y_{q}^{\prime 2}}-1=c y_{q}^{\prime}
$$

Which implies:

$$
\sqrt{1+y_{q}^{\prime 2}}=1+c y_{q}^{\prime} .
$$

This gives:

$$
1+y_{q}^{\prime 2}=1+c^{2} y_{q}^{\prime 2}+2 c y_{q}^{\prime} .
$$

Finally, we get:

$$
y_{q}^{\prime 2}\left(1-c^{2}\right)=2 c y_{q}^{\prime}
$$

Which gives: 


$$
y_{q}^{\prime}=0 \text { or } y_{q}^{\prime}=\frac{2 c}{1-c^{2}} .
$$

Let $\frac{2 c}{1-c^{2}}=A$. Then $y_{q}=A x+C$, this completes the proof.

Using Euler-Lagrange Equation (2) to find the extremals for the following function:

$$
x\left(\frac{\partial}{\partial x} y(x)\right)+\left(\frac{\partial}{\partial x} y(x)\right)^{2} .
$$

We obtain the following solution:

$$
y(x)=\frac{-1}{4} x^{2}+c_{1} x+c_{2}
$$

As q-deformation of this example, we get the following theorem.

\section{Theorem 4.2}

Let $f$ given by:

$$
f\left(t, y_{q}, y_{q}^{\prime}\right)=t y_{q}^{\prime}+y_{q}^{\prime 2}
$$

Satisfying the q-Euler Lagrange equation:

$$
D_{q, y} f-D_{q, t}\left(D_{q, y_{q}^{\prime}} f\right)=0 .
$$

Then, we get:

$$
y(t)=-\frac{1}{[2]_{q}^{2}} t^{2}+c_{1} t+c_{2} .
$$

\section{Proof}

Using Equation 5, we obtain:

$$
\begin{aligned}
& D_{q, y_{q}^{\prime}} f\left(t, y_{q}, y_{q}^{\prime}\right)=\frac{f\left(t, y_{q}, y_{q}^{\prime}\right)-f\left(t, y_{q}, q y_{q}^{\prime}\right)}{y_{q}^{\prime}(1-q)} \\
& =\frac{\left(t y_{q}^{\prime}+y_{q}^{\prime 2}\right)-\left(t q y_{q}^{\prime}+q^{2} y_{q}^{\prime 2}\right)}{y_{q}^{\prime}(1-q)} \\
& =\left(\frac{1}{1-q}\right)\left(t+y_{q}^{\prime}-q t-q^{2} y_{q}^{\prime}\right) \\
& =t+y_{q}^{\prime}\left(\frac{1-q^{2}}{1-q}\right) \\
& =t+(1+q) y_{q}^{\prime}=t+[2]_{q} y_{q}^{\prime} .
\end{aligned}
$$

Since, we have:

$$
D_{q, y} f\left(t, y_{q}, y_{q}^{\prime}\right)=0
$$

Then:

$$
D_{q, y}\left(D_{q, y_{q}^{\prime}}, f\left(t, y, y_{q}^{\prime}\right)\right)=0
$$

Therefore, we get:

$$
D_{q, y_{q}^{\prime}} f\left(t, y, y_{q}^{\prime}\right)=c
$$

This gives:

$$
t+[2]_{q} y_{q}^{\prime}=c
$$

Which is equivalent to:

$$
t+[2]_{q} \frac{y(t)-y(q t)}{t(1-q)}=c .
$$

This implies that:

$$
[2]_{q}\left(\frac{y(t)-y(q t)}{t(1-q)}\right)=c-t .
$$

Then, we get:

$$
\begin{gathered}
y(t)-y(q t)=\frac{(c-t) t(1-q)}{[2]_{q}}=\left(-t^{2}+c t\right)\left(\frac{1-q}{[2]_{q}}\right) \\
y(q t)-y\left(q^{2} t\right)=\left(-q^{2} t^{2}-c q t\right)\left(\frac{1-q}{[2]_{q}}\right) \\
\vdots \\
y\left(q^{k-1} t\right)-y\left(q^{k} t\right)=\left(-q^{2(k-1)} t^{2}-c q^{(k+1)} t\right)\left(\frac{1-q}{[2]_{q}}\right),
\end{gathered}
$$

We deduce:

$$
y(t)-y\left(q^{k} t\right)=\sum_{i=0}^{k-1}\left(-q^{2 i} t^{2}+c q^{i} t\right)\left(\frac{1-q}{[2]_{q}}\right) .
$$

But:

$$
\begin{aligned}
& \sum_{i=0}^{k-1}-q^{2 i} t^{2}=-t^{2} \sum_{i=0}^{k-1}\left(-q^{i}\right)^{i} \\
& =-t^{2}\left(\frac{1-q^{2 k}}{1-q^{2}}\right)
\end{aligned}
$$

In addition:

$$
\begin{aligned}
& \sum_{i=0}^{k-1} c q^{i} t=c t \sum_{i=0}^{k-1} q^{i} \\
& =c t\left(\frac{1-q^{t}}{1-q}\right) .
\end{aligned}
$$


Then, we get:

$$
\begin{aligned}
& y(t)=y\left(q^{k} t\right)-\frac{(1-q)}{[2]_{q}}\left(\frac{1-q^{2 k}}{1-q^{2}}\right) t^{2}+c \frac{(1-q)}{[2]_{q}}\left(\frac{1-q^{k}}{1-q}\right) t \\
& =y\left(q^{k} t\right)-\frac{\left(1-q^{2 k}\right)}{[2]_{q}^{2}} t^{2}+c \frac{\left(1-q^{k}\right)}{[2]_{q}} t .
\end{aligned}
$$

As $k \rightarrow \infty$, we obtain:

$$
\begin{aligned}
& y(t)=y(0)-\frac{1}{[2]_{q}^{2}} t^{2}+\frac{c}{[2]_{q}} t \\
& =-\frac{1}{[2]_{q}^{2}} t^{2}+c_{1} t+c_{2}
\end{aligned}
$$

where, $c_{1}=\frac{c}{[2]_{q}}$ and $c_{2}=y(0)$.

\section{Remark 2}

As $q \rightarrow \infty$, Equation (10) becomes:

$$
\begin{aligned}
& y(t)=\frac{-1}{2^{2}} t^{2}+c_{1} t+c_{2} \\
& =-\frac{1}{4} t^{2}+c_{1} t+c_{2}
\end{aligned}
$$
(8).

Which gives the classical case studied see Equation

\section{Author's Contributions}

All authors equally contributed in this work.

\section{Ethics}

This article is original and contains unpublished material. The corresponding author confirms that all of the other authors have read and approved the manuscript and no ethical issues involved.

\section{References}

Altoum, S.H., 2018a. q-deformation of transonic gas equation. J. Math. Stat., 14: 88-93. DOI: 10.3844 jmssp.2018.88.93

Altoum, S.H., 2018b. q-deformation of transonic gas equation. Am. J. Applied Sci., 15: 261-266. DOI: 10.3844/ajassp.2018.261.266

Altoum, S.H., H.A. Othman and H. Rguigui, 2017. Quantum white noise Gaussian kernel operators. Chaos Solitons Fractals, 104: 468-476.

DOI: 10.1016/j.chaos.2017.08.039

Bangerezako, G., 2004. Varitional q-calculus. J. Math. Anal. Applic., 289: 650-665.

DOI: $10.1016 /$ j.jmaa.2003.09.004

Rguigui, H., 2015a. Quantum Ornstein-uhlenbeck semigroups. Qunantum Studies: Math. Foundat., 2: 159-175. DOI: $10.1007 / \mathrm{s} 40509-014-0023-5$

Rguigui, H., 2015b. Quantum-potentials associated to quantum Ornstein - Uhlenbeck semigroups. Chaos Solitons Fractals, 73: 80-89.

Rguigui, H., 2016a. Characterization of the QWNconservation operator. Chaos Solitons Fractals, 84: 41-48. DOI: 10.1016/j.chaos.2015.12.023

Rguigui, H., 2016b. Wick differential and Poisson equations associated to the QWN-euler operator acting on generalized operators. Mathematica Slo., 66: 1487-1500. DOI: 10.1515/ms-2016-0238

Rguigui, H., 2018a. Fractional number operator and associated fractional diffusion equations. Math. Phys. Anal. Geom., 21: 1-17.

DOI: $10.1007 / \mathrm{s} 11040-017-9261-1$

Rguigui, H., 2018b. Characterization theorems for the quantum white noise gross Laplacian and applications. Complex Anal. Oper. Theory, 12: 1637-1656. DOI: 10.1007/s11785-018-0773-x 